\title{
Perfil higiênico-sanitário de um restaurante comercial no interior baiano
}

\author{
Hygienic-sanitary profile of a commercial restaurant in the interior of Bahia \\ Perfil higiénico-sanitario de un restaurante comercial en el interior de Bahía
}

\author{
Alana Oliveira Souza \\ ORCID: https://orcid.org/0000-0001-5133-2230 \\ Universidade de Pernambuco, Brasil \\ E-mail: alana.oliveirasouza@upe.br \\ Eduarda Vitória Batista Passos \\ ORCID: https://orcid.org/0000-0002-0630-9178 \\ Universidade de Pernambuco, Brasil \\ E-mail: eduarda.passos@upe.br \\ Maria Isabel Almeida Gonçalves \\ ORCID: https://orcid.org/0000-0003-3296-4763 \\ Universidade de Pernambuco, Brasil \\ E-mail: mariaisabel.goncalves@upe.br \\ Railla Lima Lacerda Coelho \\ ORCID: https://orcid.org/0000-0002-9007-9095 \\ Universidade de Pernambuco, Brasil \\ E-mail: railla.lacerda@upe.br \\ Claudileide de Sá Silva \\ ORCID: https://orcid.org/0000-0002-3677-3000 \\ Universidade de Pernambuco, Brasil \\ E-mail: Claudileide.silva@upe.br
}

\section{Resumo}

É perceptível que o consumidor se preocupa com a qualidade e higiene das preparações alimentares, visto que existem algumas ocorrências de doenças transmitidas por alimentos em estabelecimentos que comercializam alimentos prontos. Com isso, o objetivo desse artigo é avaliar as condições higiênico-sanitárias e o controle microbiológico de um restaurante localizado em Juazeiro-Ba. Para análise das condições higiênico-sanitárias do estabelecimento foi utilizada um checklist sobre boas práticas de manipulação de alimentos com base na RDC N²16/2004 ANVISA, e classificação segundo a RDC No275/2002 ANVISA. Para análise microbiológica, foram coletadas amostra de água, carne frita e de salada crua, ambas prontas para consumo, amostras de superfície do apoio de corte e do liquidificador. As amostras foram avaliadas segundo análises microbiológicas para a contagem de coliformes totais e Escherichia coli em sistema de análise Petrifilm Coliform Count Plate. Em relação às boas práticas de manipulação, o restaurante foi classificado como grupo 2 (56,5 \% adequação). Já as análises microbiológicas apontaram contaminação na salada crua por Coliformes totais ( $\left.3 \times 10^{3} \mathrm{UFC} / \mathrm{g}\right)$, sendo estes incontáveis nas amostras de água, carne frita e liquidificador. $\mathrm{O}$ apoio de corte apresentou contaminação por Coliformes totais em níveis aceitáveis para superfícies. Torna-se notório a necessidade de um responsável técnico no local a fim de obter controle higiênico-sanitário adequado, o que poderia evitar a contaminação nos alimentos e superfícies.

Palavras-chave: Boas práticas; Coliformes totais; Escherichia coli; Restaurante; Segurança e qualidade dos alimentos.

\footnotetext{
Abstract

Introduction: It is noticeable that the consumer is concerned with the quality and hygiene of food preparations, since there are some occurrences of diseases transmitted by food in establishments that sell ready-made foods. Thus, the objective of this article is to evaluate the hygienic-sanitary conditions and the microbiological control of a restaurant located in Juazeiro-Ba. Methodology: For the analysis of the hygienic-sanitary conditions of the establishment, a checklist on good food handling practices was used, based on RDC N 216 / 2004 ANVISA, and classification according to RDC No 275 / 2002 ANVISA. For microbiological analysis, water, fried meat and raw salad samples were collected, both ready for consumption, surface samples of the cutting support and blender. The samples were evaluated according to microbiological analyzes for the count of total coliforms and Escherichia coli using the Petrifilm Coliform Count Plate $3 \mathrm{M} \AA$ analysis system. Results: Regarding good handling practices, the restaurant was classified as group $2(56.5 \%$ adequacy). The microbiological analyzes showed contamination in the raw salad by total coliforms $\left(3 \times 10^{3} \mathrm{CFU} / \mathrm{g}\right)$, which are countless in the samples of water, fried meat and blender. The cutting support showed contamination by total coliforms at levels acceptable for surfaces. Conclusion: The need for an on-site
} 
technician in order to obtain adequate hygienic-sanitary control becomes evident, which could prevent contamination of food and surfaces.

Keywords: Escherichia coli; Food safety and quality; Good practices; Restaurant; Total coliforms.

\begin{abstract}
Resumen
Se nota que el consumidor se preocupa por la calidad e higiene de los preparados alimenticios, ya que existen algunas ocurrencias de enfermedades transmitidas por los alimentos en establecimientos que venden alimentos preparados. Así, el objetivo de este artículo es evaluar las condiciones higiénico-sanitarias y el control microbiológico de un restaurante ubicado en Juazeiro-Ba. Para el análisis de las condiciones higiénico-sanitarias del establecimiento se utilizó una lista de verificación de buenas prácticas de manipulación de alimentos, con base en RDC N²16 / 2004 ANVISA, y clasificación según RDC №275 / 2002 ANVISA. Para el análisis microbiológico se recogieron muestras de agua, carne frita y ensalada cruda, ambas listas para el consumo, muestras de superficie del soporte de corte y batidora. Las muestras se evaluaron de acuerdo con análisis microbiológicos para el recuento de coliformes totales y Escherichia coli utilizando la placa de recuento de coliformes Petrifilm en el sistema de análisis $3 \mathrm{M} \circledast$. En cuanto a las buenas prácticas de manejo, el restaurante se clasificó en el grupo 2 (56,5\% de adecuación). Los análisis microbiológicos mostraron contaminación en la ensalada cruda por coliformes totales ( $3 \times 10^{3} \mathrm{UFC} / \mathrm{g}$ ), los cuales son innumerables en las muestras de agua, carne frita y licuadora. El soporte de corte mostró contaminación por coliformes totales a niveles aceptables para superficies. Se evidencia la necesidad de contar con un técnico in situ para obtener un adecuado control higiénico-sanitario, que evite la contaminación de alimentos y superficies.
\end{abstract}

Palabras clave: Buenas prácticas; Calidad y seguridad alimentaria; Coliformes totales; Escherichia coli; Restaurante.

\title{
1. Introdução
}

A alimentação fora do lar é uma realidade que já se faz necessária para muitas pessoas, isso devido a distância entre o trabalho e a residência, assim como forma de lazer e socialização com amigos e parentes (Bezerra et al., 2020). No entanto, este hábito alimentar pode facilitar por um lado, porém por outro pode acarretar incertezas ao consumidor em relação a qualidade dos alimentos (Pereira et al., 2020).

A garantia da integridade de alimentos servidos em restaurantes pode evitar doenças transmitidas por alimentos (DTA's). Um surto de DTA pode acarretar danos à saúde do consumidor, bem como perdas econômicas e por consequência desvalorização do comércio frente a outros consumidores. Além de gerar desperdício de alimentos (OPAS, 2006). É fundamental que todo estabelecimento que forneça refeição possua controle higiênico-sanitário, uma vez que entre os anos de 2009 a 2018 foram registrados 6.809 surtos por DTAs, sendo a região nordeste a segunda mais prevalente, em que $16 \%$ dos surtos ocorreram em restaurantes e similares (Brasil, 2009).

Neste contexto, encontram-se aproximadamente 250 tipos de DTA`s que são responsáveis por problemas de saúde pública e perdas de alimentos com prejuízos econômicos (Melo et al, 2018). Existem diferentes agentes causadores de DTA, entre eles estão os microrganismos patogênicos e suas toxinas, agentes químicos, físicos ou biológicos. O primeiro grupo é o mais considerável, onde encontram-se uma extensa variedade de gêneros e espécies com diferentes patologias, duração de sintomas e períodos de incubação (Melo et al., 2018).

Para que o alimento seja livre de qualquer agente patogênico ou de suas toxinas, precisa-se de uma adesão à segurança primária na manipulação dos alimentos (Silva et al., 2015), e por meio das Resoluções, Portarias e leis que regulamentam a produção de refeições em serviços de alimentação, é possível obter parâmetros de controle de qualidade dos alimentos, dos manipuladores e do local a serem seguidos (Pereira et al., 2019).

Deste modo, torna-se necessário que se conheça os principais pontos que afetam a garantia do fornecimento de alimentos seguros para que seja mantida rigorosamente. Identificar e abranger esses motivos são de extrema importância na reflexão para desenvolver a motivação e aperfeiçoar práticas gerenciais. Além do que, o restaurante que oferece aos clientes a garantia do fornecimento de alimento com qualidade e eficiência tem grandes chances de continuar crescendo no mercado (Carvalho \& Mori, 2017). 
Neste sentido, a gestão da qualidade é essencial e só é possível ser por meio de documentos dentre os quais estão os procedimentos operacionais padronizados, manual de boas práticas descrito de acordo com a legislação a ser seguida e registros que comprovam a implementação dos procedimentos (Paula et al., 2017). Tendo em vista a magnitude do tema, o presente artigo tem como objetivo avaliar as condições higiênico-sanitárias e o controle microbiológico dos alimentos servidos em um restaurante localizado em Juazeiro da Bahia.

\section{Metodologia}

Trata-se de um estudo de caráter descritivo transversal, qualitativo e quantitativo, desenvolvido em novembro de 2020. A coleta de dados foi realizada em um restaurante que se encontra no centro do município de Juazeiro da Bahia, localizado no sertão do São Francisco, Brasil.

\section{Instrumento de coleta}

Inicialmente para realização da análise das condições higiênico-sanitárias do estabelecimento foi utilizada uma lista de verificação de boas práticas de manipulação de alimentos baseada nas RDC No 216/2004 da Agência Nacional de Vigilância Sanitária (ANVISA), e CVS No 5/2013, do Centro de Vigilância Sanitária do Estado de São Paulo, e classificação de acordo com RDC N 275/2002, ANVISA. Sendo assim, foram abordados 177 itens distribuídos nas seguintes seções: I) Recebimento de mercadorias; II) Armazenamento; III) Equipamentos, móveis e utensílios; IV) Instalação e edificação; V) Higiene das instalações; VI) Manipulação dos alimentos; VII) Manipuladores de alimentos; VIII) Abastecimento de água; IX) Sanitários e vestiários; X) Resíduos sólidos-lixo; XI) Área de venda e XII) Controle de vetores.

Para calcular o percentual de conformidade foi multiplicado o total de conformidades por 100 e dividido o resultado pelo total de itens indicado ao final de cada tópico analisado. Para classificações dos resultados, foram utilizados os percentuais de acordo a Resolução - RDC n 275/2002, da Agência Nacional de Vigilância Sanitária: Grupo 1 - 76 a 100\% de atendimento dos itens; Grupo 2 - 51 a 75\% de atendimento dos itens; Grupo 3 - 0 a 50\% de atendimento dos itens.

\section{Análises microbiológicas}

Para a análise microbiológica, foram coletadas: amostra de água utilizada na produção dos alimentos e higienização de utensílios; amostra de carne frita; e de salada crua, sendo ambos prontos para consumo. Além desses foram coletadas amostras de superfície do apoio de corte e do liquidificador que entram em contato direto com alimentos prontos para consumo.

As coletas das referidas amostras foram realizadas in loco, e as análises microbiológicas para a contagem de coliformes totais e Escherichia coli utilizado o sistema de análise Petrifilm Coliform Count Plate 3M®. Para classificação, foram consideradas adequadas as amostras que se encontravam abaixo do limite máximo conforme descrito na Instrução Normativa nº0 de 23 de dezembro de 2019, da ANVISA, contagem para coliformes totais conforme descrito por Freitas et al. (2020). Para as amostras de água foram utilizadas placas Petrifilm Aqua $3 \mathrm{M}{ }^{\circledR}$, por ser um sistema simplificado de análises microbiológicas para a água, e classificadas em adequadas e inadequadas segundo a Portaria de consolidação $\mathrm{n}^{\circ} 05$ de 28/09/2017 do Ministério da Saúde. Para amostras de superfície, foram consideradas satisfatórias, quando o número de colônias de coliformes totais não ultrapassou 2 por $\mathrm{cm}^{2}$ (Freitas et al., 2020).

\section{Análise estatística}

O banco de dados foi construído no Excel, versão 7.0, para Windows 10, e as análises estatísticas realizadas no software Prism, versão 5.0 (GraphPad, USA). Para tanto, utilizou-se de estatística descritiva com diferenças com valor de $\mathrm{p}$ $<0,05$, sendo considerados estatisticamente significantes. 


\section{Resultados e Discussão}

\section{Condições higiênico-sanitárias com base na lista de verificação}

O estabelecimento analisado trata-se de um restaurante no qual a refeição servida é apenas o almoço. Na Tabela 1 pode-se verificar o total de itens analisados, total de conformidades e não conformidades e o percentual de conformidades encontradas na empresa, de acordo com cada aspecto avaliado.

Tabela 1. Relação de grupo de itens avaliados através da lista de verificação quanto às boas práticas de manipulação de alimentos e seus respectivos percentuais de adequação, aplicados por um restaurante na cidade de Juazeiro-BA, 2020.

\begin{tabular}{|c|c|c|c|c|}
\hline Aspecto avaliado & $\begin{array}{c}\text { Itens } \\
\text { analisados }\end{array}$ & Conformes & Não conformes & $\begin{array}{l}\text { Percentual de } \\
\text { conformidade }\end{array}$ \\
\hline Edificações e Instalações & 50 & 26 & 19 & $52 \%$ \\
\hline Higienização & 13 & 8 & 4 & $53,33 \%$ \\
\hline $\begin{array}{l}\text { Controle Integrado de Vetores e } \\
\text { Pragas }\end{array}$ & 6 & 5 & 1 & $83,33 \%$ \\
\hline Abastecimento de Água & 12 & 7 & 1 & $58,33 \%$ \\
\hline Manejo de Resíduos & 7 & 6 & 1 & $85,71 \%$ \\
\hline Manipuladores & 17 & 16 & 1 & $94,11 \%$ \\
\hline $\begin{array}{l}\text { Matérias Primas, Ingredientes e } \\
\text { Embalagens }\end{array}$ & 17 & 7 & 7 & $41,17 \%$ \\
\hline Preparação do Alimento & 21 & 9 & 12 & $42,85 \%$ \\
\hline $\begin{array}{l}\text { Armazenamento e Transporte dos } \\
\text { Alimentos Preparados }\end{array}$ & 4 & 2 & 2 & $50 \%$ \\
\hline Exposição ao Consumo & 14 & 8 & 5 & $57,14 \%$ \\
\hline Documentação e Registro & 14 & 6 & 8 & $42,85 \%$ \\
\hline Responsabilidade técnica & 2 & 0 & 2 & $0 \%$ \\
\hline
\end{tabular}

Fonte: Autores.

Em relação a conformidade de todos os itens avaliados, o percentual médio de adequação foi de 56,5\%, classificandoo no Grupo 2. No entanto, ressalta-se que evidenciou-se um maior número de seções com percentual de adequação > 50\% (p < 0,012, teste T não pareado) do que aquelas de menor adequação.

Este resultado foi semelhante ao descrito por Carvalho et al. (2016) ao avaliar 3 restaurantes do tipo self-service no Campus da Universidade Federal do Pará, em que dois restaurantes obtiveram em média 61,4\% e 62,3\% de conformidade, se enquadrando no Grupo 2 (Regular), e um restaurante obteve 89,3\% de conformidade classificado como Grupo 1 (Bom), sendo este último o único com responsável técnico com formação superior (nutricionista).

Neste sentido, no presente estudo, o aspecto avaliado com menor adequação foi responsabilidade técnica $(0 \%)$ tendo em vista a falta de um responsável técnico no local. Com maior adequação encontram-se os itens para avaliação de manipuladores de alimentos $(94,11 \%)$, o que indica existir uma correta preocupação higiênica-sanitária com este grupo. Este dado foi interessante, pois alguns estudos relatam maior déficit de adequação para este item, como Shinohara et al. (2016) ao observar as boas práticas em serviço de alimentação e Apoli et al.(2015) ao avaliarem adoção das Boas Práticas por 
manipuladores de alimentos de restaurantes comerciais. Os citados autores verificaram que as principais não-conformidades no serviço de alimentação foram relacionadas aos manipuladores de alimentos e gestores, e cuja maioria das pessoas não possuíam domínio do conteúdo sobre a importância da higiene pessoal como fator de contaminação cruzada.

Resultado como este também foi relatado por Lima et al.(2020) ao avaliarem adequação das boas práticas de fabricação em 17 restaurantes da cidade de Dourados-MS, em que verificaram que os maiores índices de não conformidade foram encontrados nos aspectos manipuladores e documentação, em que 35\% dos restaurantes apresentaram índice igual ou superior a $50 \%$ de não conformidades para manipuladores e $65 \%$ dos restaurantes não apresentam nenhum item da documentação em conformidade. Os manipuladores de alimentos são frequentemente relacionados à contaminação dos alimentos em restaurantes devido principalmente às contaminações microbiológicas e cruzadas (Medeiros et al., 2017).

Os itens matérias-primas, ingredientes e embalagens; preparação do alimento; armazenamento e transporte dos alimentos preparados; documentação e registro merecem maior atenção por apresentarem adequação ruim $(<50 \%)$. É importante que a garantia do fornecimento de alimentos seja segura para que sejam tratados com rigor e formas adequadas, no entanto, foi possível observar que não são adotadas medidas para evitar que os insumos contaminem os alimentos preparados. Como também que o armazenamento dos ingredientes e embalagens não é limpo e organizado de forma correta; o local também não cumpre com o espaço necessário, adequado, para ventilação e limpeza; os ingredientes não são organizados em áreas protegidas e limpas, assim como os utensílios e alimentos não são higienizados de forma correta. As temperaturas não são verificadas para garantir a eficácia do tratamento térmico (cocção) e o descongelamento não é realizado sob refrigeração $\left(5^{\circ} \mathrm{C}\right)$ ou microondas (se for coccionado imediatamente após).

Com relação ao controle de pragas e o manejo de resíduos, estes itens exibiram as melhores adequações (76 a 100\%), reduzindo a probabilidade de contaminação dos alimentos por estes fatores. Porém, a higienização (53,33\% de adequação) e o preparo dos alimentos (42,85\% de adequação) foram deficitários, podendo expor os alimentos ao risco de contaminação e comprometer a saúde dos consumidores. Carlesso E Balestrin (2018) ao avaliarem condições higiênico-sanitárias de pizzarias também encontraram resultados semelhantes para higienização e preparo de alimentos. Neumann \& Fassina (2016) ao verificar as boas práticas em uma Unidade de Alimentação e Nutrição encontrou adequação de 35\% para higienização de instalações, equipamentos, móveis e utensílios, assim como 16\% para preparo de alimentos.

Desse modo, para que as boas práticas sejam aplicadas, a capacidade de segurança e qualidade dos restaurantes devem ser supervisionadas diariamente nos locais de produção e ser contínuas e cuidadosamente elaboradas para garantir a produção de alimentos inócuos. Tiboni (2017) ao avaliar as condições higiênico-sanitárias antes e após assessoria do nutricionista em um restaurante comercial verificou uma melhora significativa no estabelecimento, em termos de qualidade e segurança dos alimentos, após supervisão pelo profissional. Estes resultados reforçam a afirmativa que a supervisão por um responsável técnico, legalmente capacitado, contribui para melhor garantia de qualidade dos alimentos.

\section{Análise microbiológica}

Ao analisarmos risco de transmissão de microrganismos para os alimentos, observamos que ele possivelmente aumenta quando os controles sobre as práticas de higiene no manuseio dos alimentos são insuficientes, o que pode ser verificado no presente estudo (Tabela 1).

Conforme podemos observar ao analisarmos os resultados apontados pela lista de verificação, que estes se refletiram nos resultados das análises microbiológicas dos alimentos e superfícies (Tabela 2). 
Tabela 2. Contagem de microrganismos presentes em utensílios e alimentos de um restaurante na cidade de Juazeiro-BA, 2020.

\section{ITENS}

Escherichia coli

\section{MICRORGANISMOS}

\begin{tabular}{lcc} 
& Escherichia coli & Coliformes totais \\
\hline Apoio de corte & 0 & $0,2 \times 10 \mathrm{UCF} / \mathrm{cm}^{2}$ \\
Liquidificador & 0 & Incontável** \\
Água & 0 & Incontável** \\
Carne Frita & 0 & Incontável $* *$ \\
Salada crua & 0 & $3 \times 10^{3} \mathrm{UFC} / \mathrm{g}$ \\
\hline
\end{tabular}

**:Limites determinados pela ANVISA, IN n ${ }^{\circ}$ 60/2019:

- Escherichia coli $=10^{3} \mathrm{UFC/g}$ (hortaliças cruas); ausente em água;

- Para amostras de superfície, são consideradas satisfatória, quando o número de colônias de coliformes totais não ultrapassa 2 por $\mathrm{cm}^{2}$;

- Coliformes totais $\geq 10^{4} \mathrm{UFC} / \mathrm{g} ;{ }^{10}$ ausente em água.

Fonte: Autores.

Um exemplo deste foi em relação ao item preparo dos alimentos, que ao avaliar a amostra de salada crua, cuja contagem de coliformes totais ( 3 x $10^{3} \mathrm{UFC} / \mathrm{g}$ ) apontam para a não higienização correta dos vegetais, apesar do número de colônias apresentarem-se conforme a legislação vigente. Straccialano et al. (2016) ao analisar a qualidade microbiológica de saladas in natura servidas em restaurantes e fast foods, obtiveram um resultado de 20 amostras (83,3\%) apresentando contaminação por coliformes a $35^{\circ} \mathrm{C}$, de um total de 24 amostras avaliadas, indicando assim condições de higiene insatisfatórias. Na análise microbiológica realizada por Lima et al (2015) também foi constatada presença de coliformes nas saladas.

$\mathrm{Na}$ carne frita foram registradas incontáveis UFC de Coliformes totais, o que é indicativo das não conformidades encontradas, como não conferência da temperatura da carne na cocção, como também possível recontaminação após a cocção pela inadequada manipulação, ou mesmo más condições higiênicas-sanitárias das superfícies e utensílios que entram em contato com o alimento pronto para consumo. Tessari e Sales (2017) ao avaliar a qualidade higiênico sanitária de quibe frito verificou a presença de coliformes totais em todas as amostras, e constatou que os quibes estavam sendo comercializados fora da temperatura preconizada pela RDC 216/2004 que deve ser maior que $60^{\circ} \mathrm{C}$ e expostas no máximo por 6 horas.

Neste sentido, as mãos são um grande veículo para a transferência de agentes eventualmente patogênicos para os alimentos. Assim como o ar dos sistemas de ventilação não natural e os utensílios são também fontes comprovadas de transmissão de microorganismos (Hernández \& Tolbar, 2020). Sabe-se que é possível encontrar nas mãos mais de 150 espécies diferentes de bactérias, sendo os gêneros Streptococcus, Staphylococcus, Corynebacterium e Lactobacillus os mais frequentemente isolados (Fonseca et al., 2019). Estes fatores também podem justificar os resultados encontrados no presente estudo.

A contaminação dos alimentos durante o manuseio é uma ocorrência quando medidas higiênico-sanitárias não são adotadas e as condições ambientais são insatisfatórias para sua manipulação (Medeiros et al, 2017).

As áreas em contato com os alimentos, também são uma das maiores causas de risco na transferência de microrganismos patogênicos (Hernández \& Tobar, 2020). Com isso, foram avaliadas as superfícies do apoio de corte e liquidificador as quais entram em contato com o alimento pronto para consumo, para contagem de coliformes totais e constatou-se $0,2 \times 10 \mathrm{UCF} / \mathrm{cm}^{2}$ e incontáveis, respectivamente. Estes resultados podem ser indicativos de higienização incorreta, conforme verificado déficit de adequação para este item na lista de verificação, como também água contaminada, ou 
mesmo ambos. Fonseca et al. (2019) avaliaram a diversidade bacteriana em superfícies de restaurantes e sugeriram que a contaminação pode ocorrer durante o processo de higienização (assepsia) e preparação do alimento, salientando a importância das superfícies que entram em contato com o alimento, sem a devida desinfecção, possibilitar contaminação cruzada.

A água também pode justificar em parte estes resultados, uma vez que ao ser analisada verificou-se que a água utilizada para preparo de alimento contém incontáveis unidades formadoras de colônias de coliformes totais. Devido a quantidade e coloração apresentada pela placa Petrifilm, segundo o manual do fabricante, a água pode estar contaminada também por Pseudomonas aeruginosa. Resultado semelhante foi encontrado por Oliveira et al. (2017) ao analisarem o controle higiênico-sanitário da água em um restaurante, encontrou a presença de coliformes totais e presença de coliformes fecais/E. coli em todas as amostras coletadas, relatando assim que está não garante a qualidade dos alimentos preparados e compromete as condições higiênico-sanitárias do restaurante.

\section{Conclusão}

O presente trabalho constatou a fragilidade no controle higiênico-sanitário em um restaurante comercial, contribuindo para os achados em outras cidades brasileiras, sendo cada vez mais frequente e preocupante em virtude do aumento considerável de surtos alimentares nos últimos 5 anos.

A vigência pandêmica da COVID-19 também põe em evidência a necessidade de maior controle higiênico-sanitário dos restaurantes comerciais já despreparados em situações básicas de controle de qualidade de alimentos.

Os resultados apresentados demonstram a necessidade de melhor capacitação das equipes e o controle sério e rigoroso da higiene, preparo e conservação dos alimentos e água.

Neste sentido, torna-se notório a necessidade de um responsável técnico no local a fim de obter um controle higiênicosanitário adequado, o que poderia contribuir para o real seguimento das boas práticas de manipulação dos alimentos e com isso evitar contaminação nos alimentos e superfícies, preservando assim a saúde do cliente.

\section{Agradecimentos}

Pró-reitoria de Extensão e Cultura Programa de Fortalecimento Acadêmico - PFA, Edital PFA Extensão - 01/2019, e Pró-reitoria de Graduação Coordenação de desenvolvimento da graduação, Edital PROGRAD/PFA/UPE/Nº5/2019.

\section{Referências}

Apoli, F. P., Moser, C. S., \& Nespolo, C. R. Adoção das boas práticas por manipuladores de alimentos em restaurantes comerciais de Itaqui-RS. (2015). Anais do VII Salão Internacional de Ensino, Pesquisa e Extensão - Universidade Federal do Pampa. http://200.132.146.161/index.php/siepe/a rticle/view/16692/0.

Bezerra, A. A., Souza, E. N., Pereira, H. G. S., \& Silva, C. de S. (2020). Microbiological analysis of alfaces in crude salads marketed in commercial restaurants of the city of Petrolina, Pernambuco, Brazil. Brazilian Journal of Development, 6(12), 100252-100625. 10.34117/bjdv6n12-499

Brasil. Ministério da Saúde, Secretaria de Vigilância em Saúde, Departamento de Vigilância em Saúde, Departamento de Vigilância das Doenças Transmissíveis, Coordenação Geral de Doenças Transmissíveis. Surtos de Doenças Transmitidas por Alimentos no Brasil. https://portalarquivo s2.saude.gov.br/images/pdf/2019/fevereiro/15/Apresenta----o-Surtos-DTA---Fevereiro-2019.pdf

Carlesso, L. C., \& Balestrin, M. B. (2018). Avaliação das condições higiênico-sanitárias de pizzarias de uma cidade do meio oeste de Santa Catarina. APEvda [Internet]. https://portalperiodicos.unoesc.edu.br/apeuv/article/view/19229.

Carvalho, L. do S. da C., Ribeiro, M. do S. S., Sousa, C. L., \& Nascimento, V. H. A. do. (2016). Boas práticas e qualidade sanitária dos alimentos servidos em restaurantes do tipo self-service no Campus da Universidade Federal do Pará. Segurança Alimentar e Nutricional, 23(2), 924. 10.20396/san.v23i2.8645998

Carvalho, S. J. E., \& Mori, E. (2017). A Importância Das Boas Práticas De Manipulação Dos Alimentos Em Restaurantes: Revisão Integrativa Da Literatura. Revista E-Ciência, 5(2). 10.19095/rec.v5i2.269

Fonseca, L. C., Azevedo, G. H. M., Santana, R. M., \& Baptista, A. B. (2019). Diversidade bacteriana em superfícies de restaurantes de Palmas-TO. Universidade Federal do Tocantins. https://doi.org/10.20873/uft.2446-6492.2019v6n2p10. 
Freitas, T. P., Costa, T. D., Ximenes, G. R., Silva, C. S. (2020). Condições higiênico-sanitárias de padarias de uma cidade do sertão pernambucano. Nutrição em foco: uma abordagem holística (VOL. II). Piracanjuba-GO: Editora Conhecimento Livre.

Hernández, P. A., \& Tobar, J. A. (2019). Análisis microbiológico de superficies en contacto con alimentos. Entramado, 16(1), 240-249. 10.18041/19003803/entramado.1.6126.

Lima, E. do N. S., Mendes, R. A., do Amaral, A. B., \& Carrijo, K. de F. (2015). Análise microbiológica de saladas e água servidas em um restaurante universitário do triângulo mineiro, Minas Gerais, Brasil. Enciclopédia Biosfera, 3176-3185. 10.18677/enciclopedia_biosfera_2015_033

Medeiros, M. das G. G. de A., Carvalho, L. R. de, \& Franco, R. M. (2017). Percepção sobre a higiene dos manipuladores de alimentos e perfil microbiológico em restaurante universitário. Ciência \& Saúde Coletiva, 22(2), 383-392. 10.1590/1413-81232017222.17282015

Melo, E. S. de, Amorim, W. R. de, Pinheiro, R. E. E., Corrêa, P. G. do N., Carvalho, S. M. R. de, Santos, A. R. S. S., Sousa, F. V. de. (2018). Doenças transmitidas por alimentos e principais agentes bacterianos envolvidos em surtos no Brasil. Pubvet, 12(10), 1-9. 10.31533/pubvet.v12n10a191.1-9

Neumann L., \& Fassina P. (2016). Verificação de boas práticas em uma unidade de alimentação e nutrição de um município do vale do Taquari - RS. Revista Uningá Review, 26(1), 2178-2571. http://revista.uninga.br/index.php/uningareviews/article/view/1791.

Organização Pan-Americana de saúde. (2006). Codex Alimentarius. Higiene dos Alimentos Textos básicos. Novembro, 2020. https://iris.paho.org/há ndle/10665.2/4268

Oliveira, D. V., Leite, S. C. M., Silva, U. N. M., Medeiros, C. K. S., \& Morato, C. B. A. (2017). Diagnóstico microbiológico para controle higiênico-sanitário em um restaurante do sertão paraibano. Journal of Medicine and Health Promotion. 2(3):712-718. http://jmhp.fiponline.edu.br/pdf/cliente=130432afa10eb51d76222ce4af829a3f56.pdf.

Paula, L. N., Alves, A. R., \& Nantes, E. A. (2017). A importância do controle de qualidade na indústria do segmento alimentício. Piracanjuba: Conhecimento Online. Dezembro. https://doi.org/10.25112/rco.v2i0.1077.

Pereira, C. A., Ribeiro, N. T., Meneses, P. R., \& Pereira, C. P. (2019). Qualidade na produção de refeições de uma unidade de alimentação e nutrição (UAN) na cidade de Fortaleza-Ceará. Conexão Unifametro. https://doity.com.br/media/doity/submissoes/5da4d897-f504-4075-af4e-040343cda1d7-template-uanpdfpdf.pdf.

Pereira, H. G. de S., Souza, E. N. de, \& Silva, C. de S. (2020). Qualidade sanitária da carne caprina comercializada em feiras de uma cidade do sertão nordestino. Research, Society and Development, 9(11), 1-16.

Shinohara, N. K. S, Almeida, A. Â. M, Nunes, C. G. S. S., \& Padilha, M. R. F. (2016). Boas práticas em serviços de alimentação: não conformidades. Revista Eletrônica Diálogos Acadêmicos.10(1), 79-91.

Silva, A. A., Bassani, L, Riella, C. O., \& Antunes, M. T. (2015). Manipulação de Alimentos em uma cozinha hospitalar: Ênfase na segurança dos alimentos. Caderno pedagogia,12(1), 111-23.

Straccialano, F. F. L., Paulino, N. T. R., Braga, A. V. U., Moreli, S., Santos, R. F. S. (2016). Qualidade microbiológica de saladas in natura servidas em restaurantes e fast foods na cidade de Campinas e região. Revista Higiene Alimentar: 30(256/257),123-127.

Tessari, J., \& Sales, W. B. (2018). Avaliação da qualidade higiênico sanitária de quibe frito vendido em bares e restaurantes da região central de CuritibaParaná. Anais do EVINCI-UniBrasi; 2017; Curitiba [Internet]. Caderno de resumo: XII EVENCI, 3(1), 16-16. https://portaldeperiodicos.unibrasi 1.com.br/index.php/anaisevinci/article/view/3418.

Tiboni, G. S. (2017). Avaliação das condições higiênico-sanitárias antes e após assessoria do nutricionista em um restaurante comercial do município de Cotia, SP. Revista Higiene Alimentar: 31(274/275), 60-65. 